\title{
Finite element analysis on mechanical properties of new section of aluminium alloy members under bending moment
}

\author{
Jianbin $\mathrm{Li}^{\mathrm{a}}$, Xiawei Tan ${ }^{\mathrm{b}}$ and Zheng Zhang ${ }^{\mathrm{c}}$ \\ School of Civil Engineering, Fujian University of Technology, No.3 Xueyuan Road, \\ University Town, Fuzhou, Fujian, P.R. China, 350118

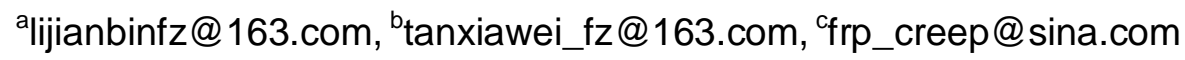

Keywords: aluminium alloy member, new section, stable performance, bending moment, FEA Abstract. Aluminium alloy structures are widely used in civil engineering. In this paper, nonlinear analyses were carried out on the aluminium alloy members under bending moments by finite element analysis. The parametric study shows that the ductility of the new section is better than that of the I-section. The stable performances of the aluminium alloy members of the new section are better than that of the I-section. The failure modes of the aluminium alloy members under bending moment are strength failure. With the type of the new section taken in this paper, the inelastic amplification factor of the members of 6061-T6 is 1.6 and that of 6061-T4 is 2.0.

\section{Introduction}

Aluminium alloy members have many advantages including high strength, fracture toughness, high fatigue strength, good corrosion resistance and good plasticity, which is why the structures are widely used in building engineering and bridge engineering. Applications in bridge structures can be traced back to the French Groslee Bridge in 1912. And in domestic civil building structures there are a large number of applications such as Tianjin Campaign Memorial in 1996, and Changsha Investment Service Center in 2005.

The section of aluminium alloy members applied widely in the aforementioned applications is the I-section. And aluminium alloy members of the I-section are assembled by welding, which weakens the strength of aluminium alloy. However, the aluminium alloy members of the new section are easier to assemble than that of the I-section from the perspective of structure's section.

The elastic modulus of aluminium alloy is only 1/3 of carbon steel [1-2]. This has caused the deformation and stability of the aluminium alloy members more prominent than steel members, which has become one of the main problems in aluminium alloy structures. With the development of general-purpose finite element software, beam189 can simulate the nonlinear analysis of the material in ANSYS. In this paper, changing the slenderness ratio and the span depth ratio is to study the influence to the ultimate load of a kind of new section, as well as the material properties.

\section{Finite element model}

The simply supported beams under the end moments were to analyze mechanical properties like Fig.1 and Fig.2.
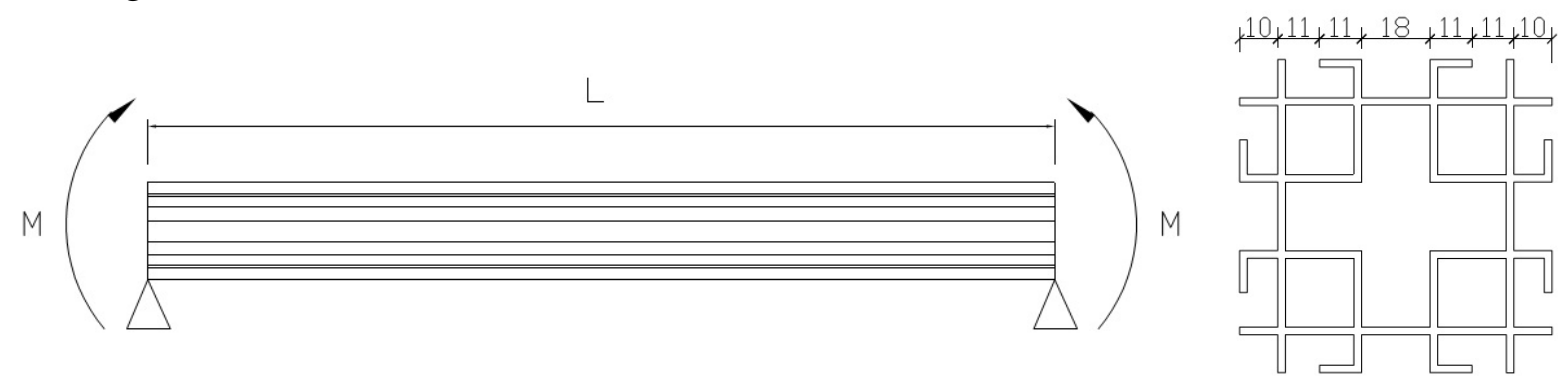

Fig. 1 Bending moment and geometrical parameters of new section 

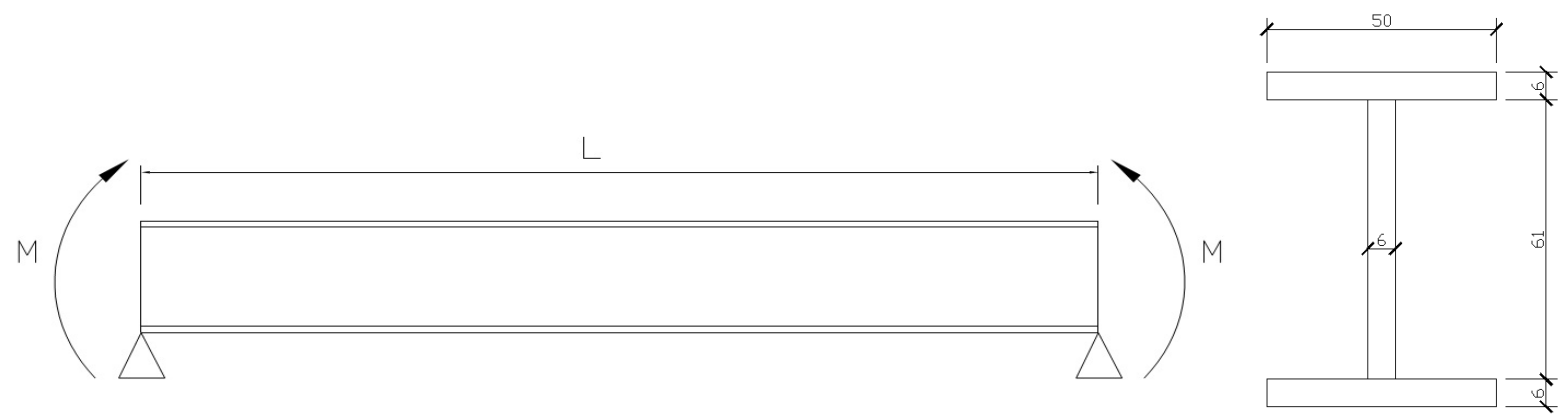

Fig. 2 Bending moment and geometrical parameters of I-section

To acquire the $M_{\mathrm{cr}} / M_{\mathrm{p}}-\lambda$ curves, the slenderness ratios were respectively $20,40,60,80,100$ and 120. So the lengths of the I-section members respectively were $0.229,0.457$ and $0.686,1.914,1.143$, $1.371 \mathrm{~m}$ and the lengths of the new section members were 0.502, 1.004, 1.506, 2.008, 2.510, $3.01 \mathrm{~m}$. To acquire the $\gamma-L / h$ curves, the span depth ratios were respectively $5,10,15,20$, and 25 . So the lengths of the new section member were $0.410,0.820,1.230,1.640,2.050 \mathrm{~m}$.

In finite element model, the study used 6061-T6 and 6061-T4 aluminium alloy, whose constitutive relationships were shown by Fig. 3. The elastic modulus of aluminium alloy was set to $70 \mathrm{GPa}$, and Poisson's ratio was set to 0.3. The nominal yield strengths of 6061-T6 and 6061-T4 aluminium alloy were $240 \mathrm{MPa}$ and $110 \mathrm{MPa}$, respectively. The stress-strain curves of aluminium alloy originated from Ramberg-Osgood model are comparable to aluminium alloys [3-4].

From the curves, there were obvious elastic stages of aluminium alloy. Compared with ordinary carbon structural steel, the structure had a long strain hardening section of aluminium alloy.

In this study, beam 189 element was used in the analysis. Finite element analysis for bucking was made of Eigenvalue analysis and load-displacement nonlinear analysis. Firstly, the buckling modes and loads was estimated, which was linear elastic analysis performed with the load applied within the step. The buckling analysis presented many buckling modes. The model accounted for the initial geometric imperfections- $L / 1000$ ( $L$ being the length of component), and only the lowest bucking mode predicted from the Eigenvalue analysis was used. Secondly, it was necessary to consider whether the post-buckling response is stable or unstable. The nonlinear material properties are incorporated in the load-displacement nonlinear analysis [5].

\section{Analysis results}

The typical failure mode was shown by Fig. 4.

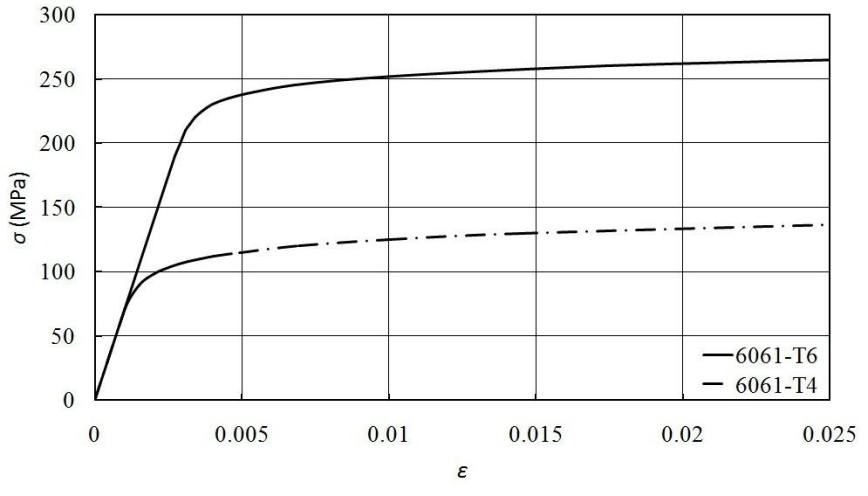

Fig. 3 Constitutive relationships

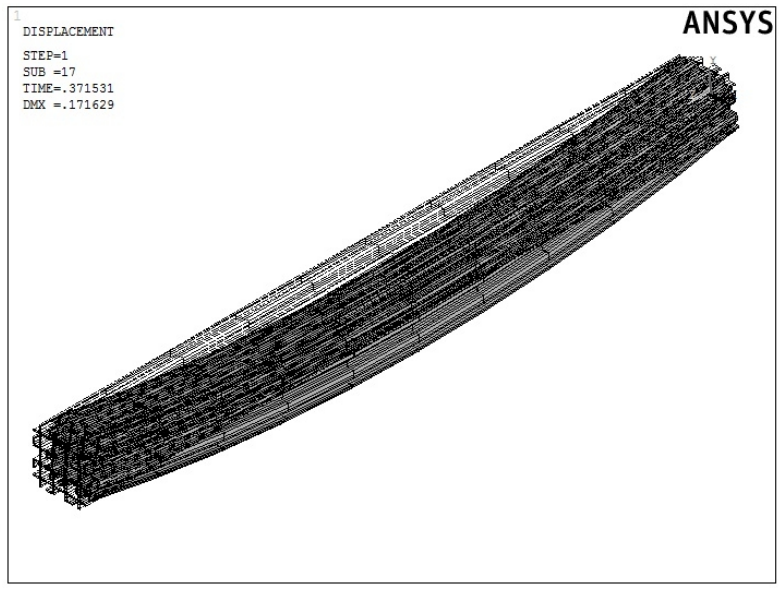

Fig. 4 Typical failure mode

The failure load is represented by $M_{x}$. The load lateral deflection is represented by $U_{y}$. The $M_{x}$ and $U_{y}$ curves of these specimens, only for slenderness ratio 60, were shown by Fig. 5 . 


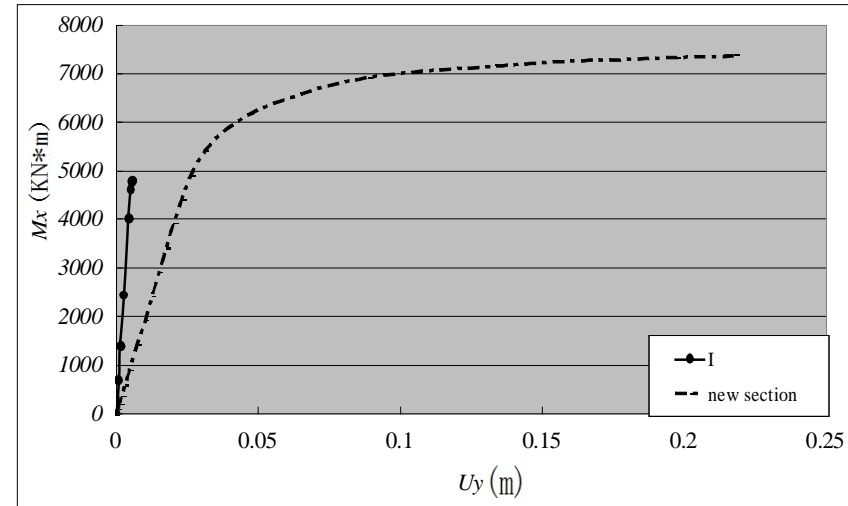

(a) 6061-T6 aluminium alloy

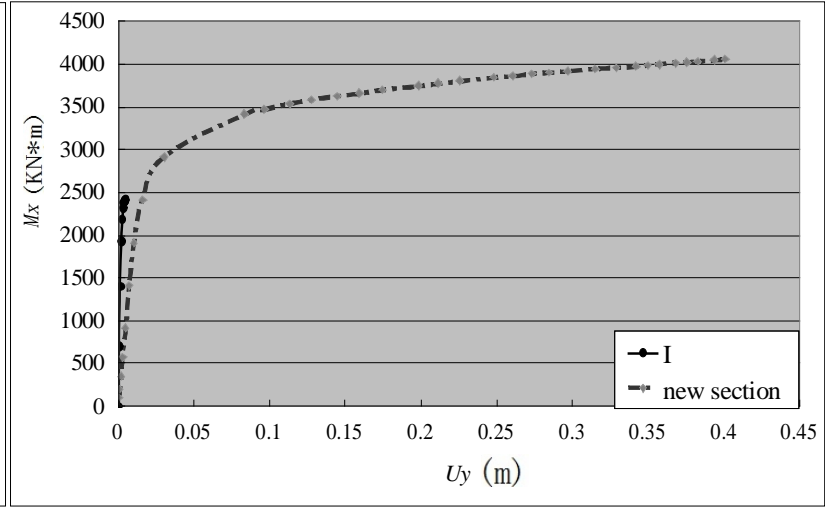

(b) 6061-T4 aluminium alloy

Fig. $5 M_{\mathrm{x}}-U_{\mathrm{y}}$ curves

From Fig. 5, it shows that the ultimate bearing capacities of the new section members are stronger than that of the I-section under the same $W_{x}$ of each other. The ultimate displacements of the new section members are much larger than that of the I-section.

The buckling factor is represented by $M_{\mathrm{cr}} / M_{\mathrm{p}}$ and the slenderness ratio is represented by $\lambda_{\mathrm{y}}$. The $M_{\mathrm{cr}} / M_{\mathrm{p}}$ and $\lambda_{\mathrm{y}}$ curves of these specimens were shown by Fig.6.

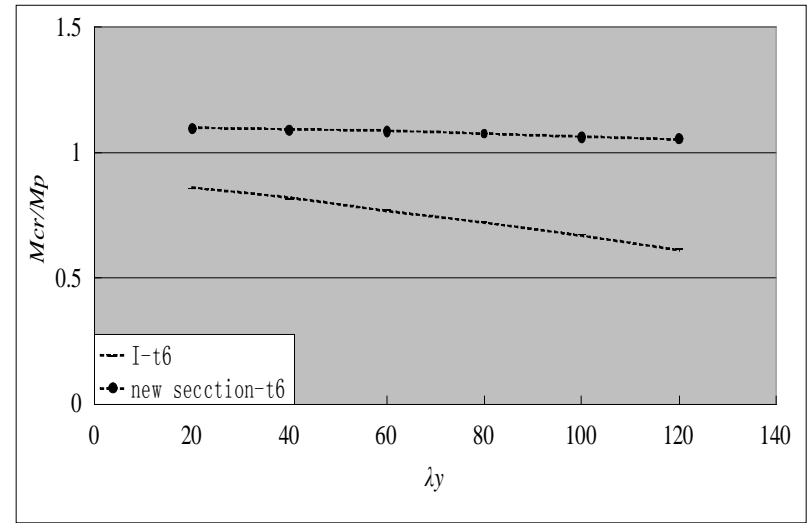

(a) 6061-T6 aluminium alloy

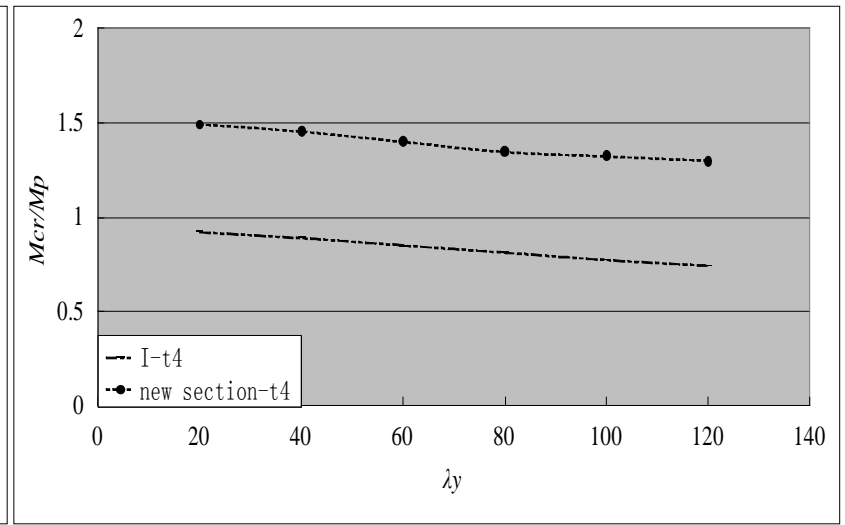

(b) 6061-T4 aluminium alloy

Fig. $6 M_{\mathrm{cr}} / M_{\mathrm{p}}-\lambda_{\mathrm{y}}$ curves

In the case of the same $W_{x}$ of each other, the curves $M_{\mathrm{cr}} / M_{\mathrm{p}}-\lambda_{\mathrm{y}}$ of new section members are higher than that of the I-section. The values of the new section's $M_{\mathrm{cr}} / M_{\mathrm{p}}$ are more than 1.0 , but that of the I-section are less than 1.0.

The inelastic amplification factor is represented by $\gamma$ and the span depth ratio is represented by $L / h$. The $\gamma$ and $L / h$ curves of these specimens were shown by Fig.7.
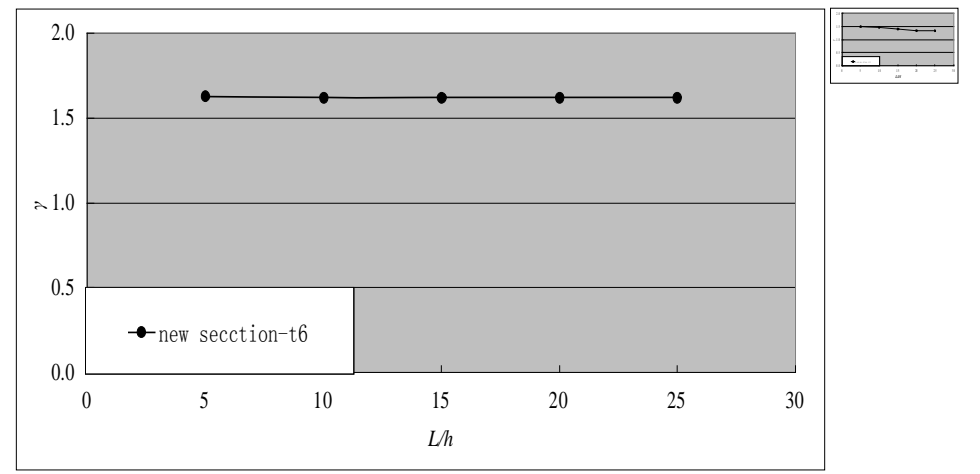

(a) 6061-T6 aluminium alloy

(b) 6061-T4 aluminium alloy

Fig. $7 \gamma$ - $L / h$ curves 
Based on the results of the finite element analysis from Fig. 7, Chinese Code for design of aluminium structures GB 50429-2007 was verified, which can be applied to the new section [6].

$$
\frac{M}{\gamma W_{x}} \leq f_{0.2}
$$

The inelastic amplification factor is represented by $\gamma$. With the type of the new section taken in this paper, the $\gamma$ of 6061-T4 is 1.6 and the $\gamma$ of 6061-T4 is 2.0. The bending moment is represented by $M$. The section module is represented by $W_{x}$. The yield stress of aluminium alloy member is represented by $f_{0.2}$.

\section{Discussion}

The mechanical properties of the new section of aluminium alloy members under the end moment have been analyzed in this paper. And the discussions are as follows:

1. In the same case of $W_{x}$, the overall stability of the aluminium alloy members of new section is higher than that of the I-section.

2. The failure modes of the new section members under bending moments are strength failure.

3. The of aluminium alloy members of the new section features higher bearing and structure ductility than that of the I-section.

4. With the type of the new section taken in this paper, the inelastic amplification factor of 6061-T6 is 1.6 and that of 6061-T4 is 2.0 .

\section{Acknowledgments}

This work was financially supported by National Natural Science Foundation of China (51208109) and Program for Outstanding Young Scientific Talents in Fujian Province University (JA13209).

\section{References}

[1] Mazzolani F.M. and Piluso V. Prediction of the Rotation Capacity of Aluminium Alloy Beams. Journal of Structural Engineering. 1999, 125: 921-929.

[2] Bradford MA, Lateral-distortional buckling of steel I-section members. Journal of Constructional Steel Research. 1992, 23: 97-116.

[3] Shen Z Y, Guo X N. Theoretical and experimental research on the aluminum alloy structure members. J Tongji Uniy (in Chinese), 2006.

[4] Zhang Q L, Zhang Z. Study on stability capacity of aluminium beam-columns. J Tongji Uniy (in Chinese), 2006.

[5] E. Ellobddy, Interaction of buckling modes in castellated steel beams. Journal of Constructional Steel Research. 2011, 67: 814-825.

[6] GB 50429-2007 Code for design of aluminium structures (in Chinese). Beijing: China Planning Press, 2008. 